\title{
Critical Node Identification for Electric Power Communication Network Based on Topology and Services Characteristics
}

\author{
Zhihui Wang ${ }^{1}$, Ran Zhang ${ }^{1,2, a}$, Xiangzhou Chen ${ }^{1}$,Yundan Liang ${ }^{3}$, Zhengdong Ren ${ }^{3}$, and Yujun $\mathrm{He}^{4}$ \\ ${ }^{1}$ China Electric Power Research Institute, Beijing 100192, China \\ ${ }^{2}$ North China Electric Power University, Beijing 102206, China \\ ${ }^{3}$ State Grid Corporation of China(SGCC), Beijing 100031, China \\ ${ }^{4}$ North China Electric Power University, Baoding 071000, China
}

\begin{abstract}
It has an important impact on the overall power communication network reliability to identify the location of critical nodes in the network and further develop protection methods. On the topology layer of power communication network, Node Topology Importance(NTI) is proposed based on calculating the rate of natural connectivity of power communication network after one node being destroyed; A calculation method of power business is presented by considering the power business requirements on the indexes, then taking into account the business layer and topology layer, weighted natural connectivity which fuses the node business information and topology information is defined, accordingly Node Combining Importance(NCI) is proposed to realize the excavation of key node. Finally, taking a real communication network as the simulating example, the simulation results by comparing with other methods show that the proposed method can improve the accuracy of recognition.
\end{abstract}

\section{Introduction}

With the rapid construction of smart grid and the continuous expansion of interconnected power grid, the safe and effective operation of the power grid is increasingly dependent on the reliable operation of the power communication network[1]. The communication equipment, that is, network node is indispensable in the power communication network business transmission. And the reliability of the power communication network will decrease immediately, even cause large-scale communication interruption once the key nodes of the network are damaged and fail. It is determined that the risk of the power grid can be reduced only if the importance of the communication network node is identified, the key nodes in the power communication network are identified and the protection is strengthened.

The normal methods for evaluating the importance of nodes include the median method, the node deletion method and the node contraction method. These normal evaluation methods are relatively single which cannot be fully applicable to a dedicated communication network with specific functional requirements such as power communication networks. It is necessary to improve the above methods or introduce new methods to evaluate the importance of nodes. According to the characteristics of the power communication network, researchers have carried out related researches: The paper [2] fused the transmission characteristics and the grid characteristics of the power communication network by using the triangular mode operators to identify the important nodes, but failed to analyze the importance of the nodes in combination with the industry characteristics of the power communication network. In the paper [3], the importance of nodes was analyzed from the special relationship between the power communication network and the power grid, and the key nodes can be identified by integrating grid impact factors and aggregation coefficients. The paper [4] defined the node importance from the static characteristics of node topology and network bandwidth, and used the position and function of the node in the power system as the weight to correct the node importance. In the paper [5], a dual-network coupling model was established based on the topological connections between the power grid and the power communication network, and a method for evaluating the importance of coupled network nodes considering cascade failure was proposed. These methods above focus on the network's topological characteristics, network bandwidth, site category and other indicators. But they don't take into account the impact of the communication business on the communication node to represent the network operation status accurately.

According to the above analysis, the concept of Node Topology Importance (NTI) based on the change of natural connectivity after node failure in the topology layer is proposed in this paper. Combined with the characteristics of electric power business, the network weighted natural connectivity is defined by fusing the network topology layer and the business layer. And the concept of the Node Combining Importance (NCI) is presented to implement the identification of the key nodes in the power communication network. The proposed method not only considers the contribution of

$\bar{a}$ Corresponding author: zrzhangran111@163.com 
nodes to the topology, but also the degree of influence of nodes on the business transmission in the network is discussed, which can be well applied to the mining of important nodes in the power communication network.

\section{Node topology importance}

When identifying the importance of a node in a network, the degree of damage to the network is usually taken into account after the node fails. The greater the damage brings, the higher the importance of the node is. The degree of damage caused by node failure to the network topology can be measured by multiple aspects, such as the reduction of the number of spanning trees and the reduction of the shortest path between nodes[6]. In fact, there is still a destructive result worth considering, that is, the reduction of alternative path redundancy in the network and the consequent decrease of the network invulnerability. From this point of view, the importance of topological layer nodes is obtained.

The metrics of the network redundant path can be represented by the natural connectivity of the network. The value of the natural connectivity is equal to the weighted sum of the number of closed paths with different lengths in the network. It can be regarded as a special form of the average eigenvalue with the clear physical meaning and concise mathematical expression, which can be derived from the adjacency matrix eigenvalue set. The power communication devices are referred to the power communication network topology diagram $G=(V, E)$ and the set of nodes $V=\left\{v_{1}, v_{2}, \ldots, v_{\mathrm{N}}\right\}$. The link between devices is defined as the edge set $E=\left\{e_{1}, e_{2}, \ldots, e_{\mathrm{M}}\right\}$. Noting $A(G)$ be the adjacency matrix of diagram $\mathrm{G}$, then the element $a_{i j}$ in $\boldsymbol{A}(G)=\left[a_{i j}\right]$ is defined as:

$a_{i j}=\left\{\begin{array}{l}0, \text { No link or link working state fails between the nodes } v_{i} \text { and } v_{j} \\ 1, \text { There are links between the nodes and the working state is normal }\end{array}\right.$

Defining $\lambda_{j}$ be the eigenvalue of the adjacency matrix $A(G)$, then the natural connectivity of the network can be denoted as[7]:

$$
\bar{\lambda}(G)=\ln \left(\frac{1}{N} \sum_{j=1}^{N} e^{\lambda_{j}(G)}\right)
$$

$\bar{\lambda}(G)$ is the natural connectivity of diagram G. The power communication network to pology subnet after the node $v_{i}$ fails is represented by $G-v_{i}$. It is assumed that all the links connected to the node fail, while the node remains in the network after the node fails in this paper. It means removing the connected links from the network, not the node. The natural connectivity of the network topology subnet $G-v_{i}$ is expressed as follows after the node $v_{i}$ fails.

$$
\bar{\lambda}\left(G-v_{i}\right)=\ln \left(\frac{1}{N} \sum_{j=1}^{N} e^{\lambda_{j}\left(G-v_{i}\right)}\right)
$$

The eigenvalue of the adjacency matrix $A\left(G-v_{i}\right)$ is represented as $\lambda_{j}\left(G-v_{i}\right)$ in this equation.

The natural connectivity is strictly monotone decreasing in regard to the deletion side. The network anti-destructive performance will deteriorate and the natural connectivity value must decrease once the node fails. The more the natural connectivity value decreases, the greater the contribution of the node to the network topology. Defining the vital function of nodes on the topology layer as Node Topology Importance(NTI). The NTI of node $v_{i}$ is expressed as:

$$
N T I_{i}=1-\frac{\bar{\lambda}\left(G-v_{i}\right)}{\bar{\lambda}(G)}
$$

In this equation, $N T I_{i}$ is the normalized importance of the node $v_{i}$. The natural connectivity of the original communication network $G$ is indicated by $\bar{\lambda}(G)$.The network topology subgraph formed after the node $v_{i}$ fails is represented by $G-v_{i} . \bar{\lambda}\left(G-v_{i}\right)$ is the natural connectivity of the network $G-v_{i}$. The higher the $N T I_{i}$ is, the greater the contribution of the node makes to the topology. Obviously, natural connectivity can be obtained by simple calculations, as well as for the largescale networks.

\section{Power communication network business importance}

The power communication network provides communication services and business supports to all aspects of the grid operation through services. According to the different ways of business attributes, security zones and service bearers, the power communication network services can be divided into different types, they have different impacts and the degree of damage on the grid in the event of interruptions or defects. And these impacts are always described by the business importance indirectly. Different types of business result in different importance. Describing and distinguishing the importance of the business accurately is the main content of the evaluation of the power communication network. The influence of the subjective factors such as the expert scoring cannot be eliminated by the existing evaluation methods. And the accuracy of the assessment of the power communication network can be affected by these subjective uncertainties. In order to overcome the subjective uncertainty, the paper [8] proposed a method to evaluate the importance of power business with business performance indicator requirements named feature index evaluation method to replace the expert subjective scoring method. It constitutes a feature indicator of the performance of the power business, mapping different requirements of different businesses to feature indicators into important value sequences and using the important value sequence of the business to build a business relative importance matrix to achieve the business importance evaluation. The feature index method proposed in the paper selects " $0,0.5,1$ " when assigning the relative importance of different services under specific performance indicators. While there are many types of business and feature indexes which make this assignment method insufficiently distinguish the importance. Based on this reason, the ratio of relative 
importance of business is further refined to make the evaluation results more reasonable in this paper.

Defining the performance indicator set $Q=\left\{Q_{l}\right\}$

$(l=1,2, \ldots, L)$, in which $Q_{l}$ represents the performance indicators of business and there are a total of $\mathrm{L}$ performance indicators; Noting the business set $S=\left\{S_{k}\right\}$

$(k=1,2, \ldots, K)$ to express the set of business types and $\mathrm{K}$ is the number of business. According to the different requirements of the performance indicators $Q_{l}$, the business set $S$ is mapped to the performance importance matrix $B_{\mathrm{kl}}=\left[1,2, \ldots, B_{l}\right]$, in which $B_{l}$ is numerically equal to the total number of different requirement levels of the indicator $Q_{l}$. Then, calculating the element $\boldsymbol{c}_{k j}^{\left(\boldsymbol{Q}_{l}\right)}$ of the business relative importance matrix $C^{\left(Q_{l}\right)}$ based on the performance importance matrix $B_{k l}$ :

$$
c_{k j}^{\left(Q_{l}\right)}=\left\{\begin{array}{cc}
1 & B_{l} \leq B_{k l} / B_{j l} \\
\vdots & \vdots \\
2 / B_{l} & 2 \leq B_{k l} / B_{j l}<3 \\
1 / B_{l} & 1 \leq B_{k l} / B_{j l}<2 \\
0 & B_{k l} / B_{j l}<1
\end{array}\right.
$$

In this equation, $\boldsymbol{c}_{k j}^{\left(\boldsymbol{Q}_{l}\right)}$ refers to the degree of importance of the business $S_{k}$ in the performance indicators $Q_{l}$ compared with the business $S_{j}$, in which $S_{k}, S_{j}(k, j=1,2, \ldots K)$ are all the services in the business set $S$. As can be seen from equation (5), ${ }^{\boldsymbol{c}_{\boldsymbol{k}}^{\left(\boldsymbol{Q}_{l}\right)}}$ has a total of $B_{l}+1$ values, that is, there are a total of $B_{l}+1$ levels for the relative importance, which are $0,1 / B_{l}, 2 / B_{l}, \ldots, 1$, and the values are related to $B_{l}$. The larger the value ${ }_{\text {of }} \boldsymbol{c}_{k j}^{\left(\boldsymbol{Q}_{l}\right)}$, the more important the service $S_{k}$ is than the service $S_{j}$ under the performance indicator $Q_{l}$. The more performance requirements of the performance indicator $Q_{l}$ as well as the level of importance, the more accurate when evaluating the business importance values. And the more the performance requirement level of the same performance indicators $Q_{l}$ and the interval is divided according to the ratio of $B_{k l}$ and $B_{j l}$, so that the more the $\boldsymbol{c}_{k j}^{\left(\boldsymbol{Q}_{l}\right)}$ value is, and the relative importance assignment of different businesses is refined under the specific performance indicators.

A comprehensive relative importance value matrix $C$ is defined whose element $c_{k j}$ is the value of the business relative importance matrix $C^{\left(Q_{l}\right)}$ summed according to the equation (6) under all the indicators:

$$
c_{k j}=\sum_{l=1}^{L} c_{k j}^{\left(B_{l}\right)}
$$

The importance values of the same business are summed under different performance indicators, that is, the elements of each row of the matrix $C$ are added to obtain the comprehensive relative importance of the business $B_{k}$ :

$$
c_{k}^{\text {sum }}=\sum_{j=1}^{K} c_{k j}
$$

$c_{k}^{\text {sum }}$ is normalized and mapped to the interval $[\mathrm{X}, 1]$, and the importance $\alpha_{k}$ of the business $S_{k}$ is obtained as:

$$
\alpha_{k}=(1-X) \frac{c_{k}^{\text {sum }}-\left(c_{k}^{\text {sum }}\right)_{\text {min }}}{\left(c_{k}^{\text {sum }}\right)_{\text {max }}-\left(c_{k}^{\text {sum }}\right)_{\text {min }}}+X
$$

In this equation, $X \in(0,1)$.

Table 1 is the business types, performance indicators and requirements selected in this paper[9]. ${ }^{c_{k j}^{\left(B_{l}\right)}}$ can be calculated from Table 1, and the business importance values of typical power communication network can be obtained from equations (6) to (8) as is shown in Table 1.

\section{Node importance combining topology and business}

The node importance is the main basis to identify the key nodes. Most of the existing methods for evaluating the node importance of power communication network are to consider the network topology or bandwidth traffic. The types and features of businesses carried by the power communication network have industry characteristics. When evaluating the node importance, not only the topology but also the importance and traffic of the businesses carried by the node should be considered[10]. So it is of more practical significance to study the key nodes of the power communication network by combining the business characteristics and topologies.

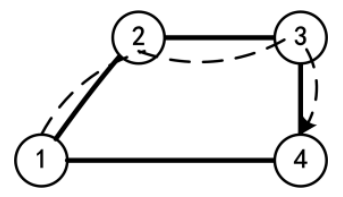

Figure. 1 A simple topology of power communication network

Link is the physical channel between nodes whose business load directly reflects the business load situation of nodes. The coupling degree of bearing relationship $d_{i j p}$ between the link of the nodes $v_{i}$ and $v_{j}$ and business $p$ is divided into polar coupling, general coupling and non-coupling. Therefore, $d_{i j p}$ has three values represented by $1,0.5$ and 0 : When the routing of business $p$ passes through the link between the nodes $v_{i}$ and $v_{j}$, and the nodes $v_{i}$ and $v_{j}$ are the source nodes or sink nodes of business $p$, the link is coupled with the business and $d_{i j p}=1$; And when the business $p$ passes through the link between the nodes $v_{i}$ and $v_{j}$, and neither of the two nodes is the source node or sink node, the link is generally coupled with the business and $d_{i j p}=0.5$; While the business $p$ does not go through the link between the nodes $v_{i}$ and $v_{j}$, the link is not coupled with the business and $d_{i j p}=0$. The simple topology shown in Figure 1 will be illustrated. The dotted line in the network is a business 
Table 1 Selected power business property indexes and their relative importance

\begin{tabular}{|c|c|c|c|c|c|c|}
\hline \multirow{2}{*}{$\begin{array}{c}\text { Power } \\
\text { communication } \\
\text { business / Label }\end{array}$} & \multicolumn{5}{|c|}{ Performance indicator requirement / Important level } & \multirow[b]{2}{*}{$\begin{array}{l}\text { Business } \\
\text { importance }\end{array}$} \\
\hline & Business channel & Load mode & $\begin{array}{l}\text { Safety } \\
\text { zone }\end{array}$ & Delay & $\begin{array}{l}\text { BER: } \\
\text { Biterror }\end{array}$ & \\
\hline $\begin{array}{l}500 \mathrm{kV} \text { relay } \\
\text { protection } / \mathrm{S}_{1}\end{array}$ & $500 \mathrm{kV}$ and above substation $/ 7$ & Dedicated channel /6 & I Zone /4 & $\leq 7 \mathrm{~ms} / 8$ & $\leq 10^{-6} / 3$ & 1.0000 \\
\hline $\begin{array}{l}220 \mathrm{kV} \text { relay } \\
\text { protection } / \mathrm{S}_{2}\end{array}$ & $220 \mathrm{kV}$ and above substation $/ 6$ & Dedicated channel /6 & I Zone /4 & $\leq 7 \mathrm{~ms} / 8$ & $\leq 10^{-6} / 3$ & 0.9224 \\
\hline Security system $/ \mathrm{S}_{3}$ & $\begin{array}{l}\text { Provincial dispatching - } \\
\text { Substation; } \\
\text { Provincial dispatching - } \\
\text { Regional dispatching /3 }\end{array}$ & Dedicated channel $/ 6$ & I Zone $/ 4$ & $\leq 25 \mathrm{~ms} / 7$ & $\leq 10^{-7} / 4$ & 0.8609 \\
\hline $\begin{array}{l}\text { Dispatching } \\
\text { telephone } / \mathrm{S}_{4}\end{array}$ & $\begin{array}{l}\text { Provincial dispatching - } \\
\text { Substation /Power plant /4 }\end{array}$ & $\begin{array}{c}\text { Dispatching switch } \\
\text { network } \text { 、 dispatching data } \\
\text { network } / 3\end{array}$ & I Zone /4 & $\leq 142 \mathrm{~ms} / 5$ & $\leq 10^{-3} / 1$ & 0.5797 \\
\hline $\begin{array}{l}\text { Dispatching } \\
\text { automation } / \mathrm{S}_{5}\end{array}$ & $\begin{array}{l}\text { Provincial dispatching - } \\
\text { Substation; } \\
\text { Provincial dispatching - } \\
\text { Regional dispatching /3 }\end{array}$ & $\begin{array}{c}\text { Dedicated channel、 } \\
\text { dispatching data network } / 5\end{array}$ & I Zone /4 & $\leq 94 \mathrm{~ms} / 6$ & $\leq 10^{-6} / 3$ & 0.6385 \\
\hline $\begin{array}{c}\text { Protection } \\
\text { information } \\
\text { management } / \mathrm{S}_{6}\end{array}$ & $\begin{array}{l}\text { Provincial and municipal } \\
\text { substation } / 5\end{array}$ & Dispatching data network /4 & II Zone $/ 3$ & $\leq 12 \mathrm{~min} / 2$ & $\leq 10^{-5} / 2$ & 0.4538 \\
\hline $\begin{array}{l}\text { Wide-area phase } \\
\text { measurement } / \mathrm{S}_{7}\end{array}$ & $\begin{array}{c}\text { provincial dispatching - } \\
\text { Substation } / 4\end{array}$ & Dispatching data network /4 & I Zone $/ 4$ & $\leq 25 \mathrm{~ms} / 7$ & $\leq 10^{-9} / 5$ & 0.7748 \\
\hline $\begin{array}{l}\text { Lightning location } \\
\text { monitoring } / \mathrm{S}_{8}\end{array}$ & $\begin{array}{c}\text { Monitoring center - Monitoring } \\
\text { station } / 1\end{array}$ & Integrated data network /2 & III Zone $/ 2$ & $\leq 240 \mathrm{~ms} / 4$ & $\leq 10^{-5} / 2$ & 0.2770 \\
\hline $\begin{array}{l}\text { Substation video } \\
\text { surveillance } / \mathrm{S}_{9}\end{array}$ & $\begin{array}{c}\text { Provincial dispatching - } \\
\text { Substation; } \\
\text { Provincial dispatching - } \\
\text { Regional dispatching /3 }\end{array}$ & Integrated data network $/ 2$ & III Zone $/ 2$ & $\leq 142 \mathrm{~ms} / 5$ & $\leq 10^{-3} / 1$ & 0.2871 \\
\hline $\begin{array}{l}\text { Video conference } \\
\qquad / \mathrm{S}_{10}\end{array}$ & $\begin{array}{l}\text { General dispatching - } \\
\text { Provincial dispatching / } \\
\text { Regional dispatching /2 }\end{array}$ & $\begin{array}{c}\text { Dedicated channel / } \\
\text { Integrated data network } / 3\end{array}$ & IV Zone /1 & $\leq 7 \mathrm{~ms} / 8$ & $\leq 10^{-5} / 2$ & 0.3665 \\
\hline $\begin{array}{l}\text { Executive } \\
\text { telephone } / \mathrm{S}_{11}\end{array}$ & $\begin{array}{l}\text { Dispatching center and plant } \\
\text { station } / 4\end{array}$ & $\begin{array}{l}\text { Integrated data network / } \\
\text { Executive exchange / } 1\end{array}$ & IV Zone /1 & $\leq 7 \mathrm{~ms} / 8$ & $\leq 10^{-3} / 1$ & 0.2238 \\
\hline $\begin{array}{l}\text { Office automation } \\
\qquad / \mathrm{S}_{12}\end{array}$ & $\begin{array}{l}\text { General dispatching - } \\
\text { Provincial dispatching / } \\
\text { Regional dispatching /2 }\end{array}$ & Integrated data network / 2 & IV Zone $/ 1$ & $\begin{array}{l}\text { No specific } \\
\text { requirement /1 }\end{array}$ & $\leq 10^{-}$ & 0.0940 \\
\hline
\end{tabular}

path $\mathrm{p}$ and in which the nodes $v_{l}$ and $v_{4}$ are the source nodes or sink nodes, and the business path goes through the nodes $v_{2}$ and $v_{3}$. Then the links between the nodes $v_{l}$, $v_{2}$ and the nodes $v_{3}, v_{4}$ are coupled with the business, and $d_{12 p}=d_{34 p}=1$; The links between the nodes $v_{2}$ and $v_{3}$ are generally coupled with business, and $d_{23 p}=0.5$; And the links between the nodes $v_{l}$ and $v_{4}$ are not coupled with business, and $d_{14 p}=0$.

The business weight of business $p$ is represented as $\gamma_{p}$, then the element $w_{i j}$ of the business weight matrix $W(G)$ of the link is as follows:

$$
w_{i j}=\sum_{p=1}^{P} d_{i j p} \gamma_{p}
$$

$$
\text { In this equation, } \gamma_{p}=\sum_{k=1}^{n_{p}} \alpha_{k} \beta_{p k},
$$
, in which np refers to the number of business types loaded between the $p$-th source and sink node pairs, and $\alpha_{k}$ represents the importance value of business $B_{k}$, while the number of business $B_{k}$ runs between the $p$-th source and sink node pairs is represented as $\beta_{p k}$.
The non-zero elements of the adjacency matrix are all 1 , while the element wij of the business weight matrix is easy to ignore the influence of topology characteristics on the importance evaluation of nodes based on its various values and large differences. Then $w_{i j}$ is normalized. The normalization function is as follows:

$$
h_{i j}=\frac{w_{i j}}{\max _{i, j=1}^{N}\left\{w_{i j}\right\}}
$$

$h_{i j}$ is used as the element corresponding to the position of the weighted adjacency matrix $H(G)$. It can be seen from equations (9) and (10) that $h_{i j}$ represents the normalized business weight value of the link between the nodes $v_{i}$ and $v_{j}$.

In the topology, the impact of the middle node on the network invulnerability reflected by the natural connectivity can reasonably evaluate the node topology importance[11]. Assuming that the eigenvalue of the weighted adjacency matrix $H(G)$ is $\lambda_{j}^{\prime}(\mathrm{G})$. Then the definition of the weighted natural connectivity of the 
power communication network is given by combining the topology and business characteristics in this paper.

$$
\bar{\lambda}^{\prime}(G)=\ln \left(\frac{1}{N} \sum_{j=1}^{N} e^{\lambda_{j}^{\prime}(G)}\right)
$$

The topology of the power communication network and the transmission of network services will be damaged by the failure of node $v_{i}$. And the value of the weighted natural connectivity of the power communication network becomes smaller. The Node Combining Importance(NCI) can be obtained by calculating the change of the weighted natural connectivity of the network before and after the failure of the node. The expression is as in equation (12).

$$
N C I_{i}=1-\frac{\overline{\lambda^{\prime}}\left(G-v_{i}\right)}{\overline{\lambda^{\prime}}(G)}
$$

In this equation, $N C I_{i}$ is the normalized combining importance of node $v_{i}$; The weighted natural connectivity of the original network topology graph $\mathrm{G}$ is represented as $\overline{\lambda^{\prime}}(G)$; And $\overline{\lambda^{\prime}}\left(G-v_{i}\right)$ is defined as the weighted natural connectivity of the subnet $\mathrm{G}-v_{i}$. The smaller the $\overline{\lambda^{\prime}}\left(G-v_{i}\right)$ is, the larger the $N C I_{i}$ can we get, and the more important the node $v_{i}$ is.

The Node Combining Importance (NCI) takes into account the node topology characteristics and power service characteristics comprehensively[12]. While reflecting the impact of the node on the invulnerability of the topology layer, combining the contribution degree of the business layer to the business operation status at the same time. Thus the key node can be identified more accurately.

\section{The case analysis}

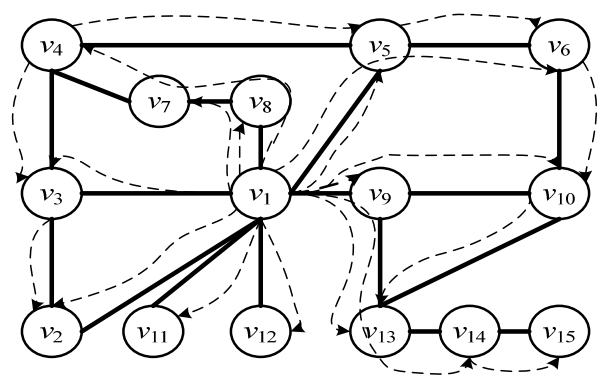

Figure. 2 Topology and routing distribution of a power communication network

A power communication network and business path model are shown in Figure 2. The network contains 15 nodes and 19 links, and the dotted lines in the network represent the business path[13]. Abstractly, the provincial dispatching is the node $v_{l}$, the regional dispatching is expressed as the node $v_{14}$, the $500 \mathrm{kV}$ substations are nodes $v_{5}$ and $v_{9}$, and the $220 \mathrm{kV}$ substations are all represented as other nodes. The business types and numbers loaded by node pairs are shown in Table 2.

\begin{tabular}{|c|c|}
\hline $\begin{array}{l}\text { Node } \\
\text { pair }\end{array}$ & Business type $\times$ number \\
\hline$(V 1, V 2)$ & $S_{3} \times 3+S_{4} \times 3+S_{5} \times 3+S_{6} \times 1+S_{7} \times 3+S_{8} \times 2+S_{9} \times 1+S_{10} \times 1+S_{11} \times 1+S_{12} \times 2$ \\
\hline$(V 1, V 3)$ & $S_{3} \times 5+S_{4} \times 4+S_{5} \times 8+S_{6} \times 2+S_{7} \times 6+S_{8} \times 8+S_{9} \times 2+S_{10} \times 4+S_{11} \times 1+S_{12} \times 4$ \\
\hline$(V 1, V 4)$ & $S_{3} \times 5+S_{4} \times 4+S_{5} \times 6+S_{6} \times 2+S_{7} \times 4+S_{8} \times 4+S_{9} \times 2+S_{10} \times 4+S_{11} \times 1+S_{12} \times 4$ \\
\hline$(v 1, V 5)$ & $S_{3} \times 5+S_{4} \times 4+S_{5} \times 6+S_{6} \times 2+S_{7} \times 6+S_{8} \times 7+S_{9} \times 2+S_{10} \times 4+S_{11} \times 1+S_{12} \times 4$ \\
\hline$(V 1, V 6)$ & $S_{3} \times 4+S_{4} \times 4+S_{5} \times 8+S_{6} \times 2+S_{7} \times 6+S_{8} \times 7+S_{9} \times 2+S_{10} \times 4+S_{11} \times 1+S_{12} \times 4$ \\
\hline$(v 1, v 7)$ & $S_{3} \times 3+S_{4} \times 3+S_{5} \times 2+S_{6} \times 2+S_{7} \times 4+S_{8} \times 2+S_{9} \times 2+S_{10} \times 4+S_{11} \times 1+S_{12} \times 4$ \\
\hline$(v 1, v 8)$ & $S_{3} \times 2+S_{4} \times 2+S_{5} \times 5+S_{6} \times 1+S_{7} \times 2+S_{8} \times 3+S_{9} \times 1+S_{10} \times 1+S_{11} \times 2+S_{12} \times 4$ \\
\hline$(V 1, V q)$ & $S_{3} \times 6+S_{4} \times 5+S_{5} \times 10+S_{6} \times 6+S_{7} \times 9+S_{8} \times 4+S_{9} \times 1+S_{10} \times 3+S_{11} \times 5+S_{12} \times 2$ \\
\hline$(V 1, v 10)$ & $S_{3} \times 5+S_{4} \times 5+S_{5} \times 9+S_{6} \times 6+S_{7} \times 8+S_{8} \times 4+S_{9} \times 1+S_{10} \times 3+S_{11} \times 4+S_{12} \times 2$ \\
\hline$(V 1, V 11)$ & $S_{3} \times 4+S_{4} \times 3+S_{5} \times 5+S_{6} \times 2+S_{7} \times 4+S_{8} \times 3+S_{9} \times 2+S_{10} \times 1+S_{11} \times 2+S_{12} \times 2$ \\
\hline$(V 1, V 12)$ & $S_{3} \times 4+S_{4} \times 4+S_{5} \times 6+S_{6} \times 2+S_{7} \times 5+S_{8} \times 5+S_{9} \times 2+S_{10} \times 1+S_{11} \times 4+S_{12} \times 1$ \\
\hline$(V 1, V 13)$ & $S_{3} \times 2+S_{4} \times 2+S_{5} \times 3+S_{6} \times 1+S_{7} \times 3+S_{8} \times 1+S_{9} \times 2+S_{10} \times 2+S_{11} \times 2+S_{12} \times 1$ \\
\hline$(V 1, V 14)$ & $S_{3} \times 4+S_{5} \times 7+S_{8} \times 2+S_{9} \times 3+S_{10} \times 2+S_{11} \times 2+S_{12} \times 1$ \\
\hline$(V 3, V 2)$ & $S_{1} \times 1+S_{8} \times 1+S_{11} \times 1$ \\
\hline$(V 4, V 3)$ & $S_{1} \times 1+S_{8} \times 1+S_{11} \times 1$ \\
\hline$(V 4, V 5)$ & $S_{1} \times 1+S_{8} \times 1+S_{11} \times 1$ \\
\hline$(v 5, V 6)$ & $S_{1} \times 1+S_{8} \times 1+S_{11} \times 1$ \\
\hline$(\mathrm{V} 6, \mathrm{~V} 10)$ & $S_{1} \times 1+S_{8} \times 1+S_{11} \times 1$ \\
\hline$(V 10, V 13)$ & $S_{1} \times 1+S_{8} \times 1+S_{11} \times 1$ \\
\hline$(V 14, V 15)$ & $S_{3} \times 1+S_{4} \times 1+S_{5} \times 1+S_{6} \times 2+S_{7} \times 1+S_{8} \times 2+S_{10} \times 1+S_{11} \times 1+S_{12} \times 2$ \\
\hline
\end{tabular}

Table 2 Business type and number between node pairs

The node set of the network model in Figure 2 is $V=\left\{v_{1}, v_{2}, v_{3}, v_{4}, v_{5}, v_{6}, v_{7}, v_{8}, v_{9}, v_{10}, v_{11}, v_{12}, v_{13}, v_{14}, v_{15}\right\}$. And the adjacency matrix $A(G)$ is obtained from the network topology:

$A(G)=\left[\begin{array}{lllllllllllllll}0 & 1 & 1 & 0 & 1 & 0 & 0 & 1 & 1 & 0 & 1 & 1 & 0 & 0 & 0 \\ 1 & 0 & 1 & 0 & 0 & 0 & 0 & 0 & 0 & 0 & 0 & 0 & 0 & 0 & 0 \\ 1 & 1 & 0 & 1 & 0 & 0 & 0 & 0 & 0 & 0 & 0 & 0 & 0 & 0 & 0 \\ 0 & 0 & 1 & 0 & 1 & 0 & 1 & 0 & 0 & 0 & 0 & 0 & 0 & 0 & 0 \\ 1 & 0 & 0 & 1 & 0 & 1 & 0 & 0 & 0 & 0 & 0 & 0 & 0 & 0 & 0 \\ 0 & 0 & 0 & 0 & 1 & 0 & 0 & 0 & 0 & 1 & 0 & 0 & 0 & 0 & 0 \\ 0 & 0 & 0 & 1 & 0 & 0 & 0 & 1 & 0 & 0 & 0 & 0 & 0 & 0 & 0 \\ 1 & 0 & 0 & 0 & 0 & 0 & 1 & 0 & 0 & 0 & 0 & 0 & 0 & 0 & 0 \\ 1 & 0 & 0 & 0 & 0 & 0 & 0 & 0 & 0 & 1 & 0 & 0 & 1 & 0 & 0 \\ 0 & 0 & 0 & 0 & 0 & 1 & 0 & 0 & 1 & 0 & 0 & 0 & 1 & 0 & 0 \\ 1 & 0 & 0 & 0 & 0 & 0 & 0 & 0 & 0 & 0 & 0 & 0 & 0 & 0 & 0 \\ 1 & 0 & 0 & 0 & 0 & 0 & 0 & 0 & 0 & 0 & 0 & 0 & 0 & 0 & 0 \\ 0 & 0 & 0 & 0 & 0 & 0 & 0 & 0 & 1 & 1 & 0 & 0 & 0 & 1 & 0 \\ 0 & 0 & 0 & 0 & 0 & 0 & 0 & 0 & 0 & 0 & 0 & 0 & 1 & 0 & 1 \\ 0 & 0 & 0 & 0 & 0 & 0 & 0 & 0 & 0 & 0 & 0 & 0 & 0 & 1 & 0\end{array}\right]$

The set of the power business carried is $S=\left\{S_{1}, S_{2}, S_{3}, S_{4}, S_{5}, S_{6}, S_{7}, S_{8}, S_{9}, S_{10}, S_{11}, S_{12}\right\}$

According to the business path shown by the dotted line in Figure 2 and the business types and numbers between the nodes given in Table 2[14], the weighted adjacency matrix $H(G)$ of network obtained by the equation (10) is as: 


$H(G)=\left[\begin{array}{ccccccccccccccc}0 & 0.286 & 0.576 & 0 & 0.807 & 0 & 0 & 0.669 & 1.000 & 0 & 0.397 & 0.475 & 0 & 0 & 0 \\ 0.286 & 0 & 0.039 & 0 & 0 & 0 & 0 & 0 & 0 & 0 & 0 & 0 & 0 & 0 & 0 \\ 0.576 & 0.039 & 0 & 0.039 & 0 & 0 & 0 & 0 & 0 & 0 & 0 & 0 & 0 & 0 & 0 \\ 0 & 0 & 0.039 & 0 & 0.039 & 0 & 0.235 & 0 & 0 & 0 & 0 & 0 & 0 & 0 & 0 \\ 0.807 & 0 & 0 & 0.039 & 0 & 0.311 & 0 & 0 & 0 & 0 & 0 & 0 & 0 & 0 & 0 \\ 0 & 0 & 0 & 0 & 0.311 & 0 & 0 & 0 & 0 & 0.039 & 0 & 0 & 0 & 0 & 0 \\ 0 & 0 & 0 & 0.235 & 0 & 0 & 0 & 0.397 & 0 & 0 & 0 & 0 & 0 & 0 & 0 \\ 0.669 & 0 & 0 & 0 & 0 & 0 & 0.397 & 0 & 0 & 0 & 0 & 0 & 0 & 0 & 0 \\ 1.000 & 0 & 0 & 0 & 0 & 0 & 0 & 0 & 0 & 0.155 & 0 & 0 & 0.273 & 0 & 0 \\ 0 & 0 & 0 & 0 & 0 & 0.039 & 0 & 0 & 0.155 & 0 & 0 & 0 & 0.039 & 0 & 0 \\ 0.397 & 0 & 0 & 0 & 0 & 0 & 0 & 0 & 0 & 0 & 0 & 0 & 0 & 0 & 0 \\ 0.475 & 0 & 0 & 0 & 0 & 0 & 0 & 0 & 0 & 0 & 0 & 0 & 0 & 0 & 0 \\ 0 & 0 & 0 & 0 & 0 & 0 & 0 & 0 & 0.273 & 0.039 & 0 & 0 & 0 & 0.142 & 0 \\ 0 & 0 & 0 & 0 & 0 & 0 & 0 & 0 & 0 & 0 & 0 & 0 & 0.142 & 0 & 0.137 \\ 0 & 0 & 0 & 0 & 0 & 0 & 0 & 0 & 0 & 0 & 0 & 0 & 0 & 0.137 & 0\end{array}\right]$

The NTI and NCI values of each node can be calculated through the matlab simulation. Taking the node deletion method (method 1) and the node contraction method (method 2) as the comparison algorithms to verify the validity and accuracy of the method proposed in this paper[15]. And programming to achieve each algorithm simulation. Table 3 lists each algorithm in accordance with the NCI algorithm ranking.

Table 3 The ranking result of the value of node importance in Figure. 2

\begin{tabular}{ccccccccc}
\hline & \multicolumn{2}{c}{ Method 1 } & \multicolumn{2}{c}{ Method 2 } & NTI algorithm & NCI algorithm \\
\cline { 2 - 8 } Node & $\begin{array}{c}\text { Importance } \\
\text { value }\end{array}$ & Rank & $\begin{array}{c}\text { Importance } \\
\text { value }\end{array}$ & Rank & NTI & Rank & NCI & Rank \\
\hline$v_{1}$ & 1.000 & 1 & 0.584 & 1 & 0.359 & 1 & 0.876 & 1 \\
$v_{9}$ & 0.923 & 6 & 0.367 & 2 & 0.150 & 3 & 0.348 & 2 \\
$v_{5}$ & 0.925 & 4 & 0.251 & 6 & 0.130 & 4 & 0.236 & 3 \\
$v_{8}$ & 0.741 & 10 & 0.010 & 12 & 0.079 & 9 & 0.185 & 4 \\
$v_{3}$ & 0.880 & 8 & 0.237 & 7 & 0.165 & 2 & 0.111 & 5 \\
$v_{12}$ & 0.000 & 13 & 0.069 & 14 & 0.047 & 13 & 0.074 & 6 \\
$v_{11}$ & 0.000 & 13 & 0.069 & 14 & 0.047 & 13 & 0.052 & 7 \\
$v_{7}$ & 0.741 & 10 & 0.010 & 12 & 0.068 & 11 & 0.049 & 8 \\
$v_{2}$ & 0.627 & 12 & 0.128 & 11 & 0.118 & 7 & 0.029 & 9 \\
$v_{13}$ & 1.000 & 1 & 0.368 & 2 & 0.118 & 6 & 0.024 & 10 \\
$v_{6}$ & 0.770 & 9 & 0.176 & 9 & 0.062 & 10 & 0.024 & 11 \\
$v_{4}$ & 0.925 & 4 & 0.237 & 7 & 0.109 & 8 & 0.013 & 12 \\
$v_{14}$ & 1.000 & 1 & 0.276 & 5 & 0.050 & 12 & 0.008 & 13 \\
$v_{10}$ & 0.923 & 7 & 0.313 & 4 & 0.121 & 5 & 0.007 & 14 \\
$v_{15}$ & 0.000 & 13 & 0.155 & 10 & 0.022 & 15 & 0.004 & 15 \\
\hline & & & & & & & &
\end{tabular}

As it can be seen from the simulation results, among the top seven nodes, the coincidence rate of NTI and the node contraction method are as high as $71.4 \%$. Meanwhile, the evaluation results of both algorithms are that node $v_{l}$ has the highest degree of importance, and the nodes $v_{11}$ and $v_{12}$ are ranked lower, indicating the effectiveness of NTI. If the network is not connected after the node fails, then the node deletion method is unavailable and the method fails[16]. While the NTI algorithm does not require the network connectivity after the node fails, which effectively overcomes the limitation of the node deletion method. Another disadvantage of the node deletion method is the inability to distinguish the importance of all nodes in the network. For example, the importance of nodes $v_{1}, v_{13}$ and $v_{14}$ calculated by the node deletion method is 1 , which is not consistent with the actual situation of the network.
However, the importance of all nodes in the network can be completely distinguished according to the NTI algorithm. In the experimental results in Table 3 , the importance of nodes $v_{7}, v_{8}$ and nodes $v_{9}, v_{13}$ cannot be distinguished by the node contraction method, while NTI can distinguish the importance of the two groups of nodes, indicating that the NTI evaluation has higher accuracy[17].

The node importance obtained by the NCI algorithm is quite different from the calculation results obtained by the other three algorithms from the comprehensive analysis of the data in Table 3. The NCI algorithm not only takes the network topology into account, but also the business characteristics loaded by the nodes. The importance of node $v_{l}$ is much higher than that of other nodes, and this result highlights the central position and the important role of node $v_{l}$ as a provincial dispatching. Comparing the four algorithms, it can be seen that NCI significantly improves the importance ranking of nodes directly connected to node $v_{1}$, such as nodes $v_{5}, v_{8}, v_{11}$ and $v_{12}$. Because these nodes are necessary for the provincial dispatching nodes and some other nodes to transmit business, which carrying a large number of important services, being of high importance and conforming to the actual situation of the power communication network. It indicates the rationality of the NCI. NTI and NCI reduce the importance of node $v_{14}$, because node $v_{14}$ is at the edge of the topology and transmits less business. From the above, the node importance ranking obtained by the NCI algorithm proposed in this paper is closer to the actual operation of the power communication network, which can effectively identify the key sites and reduce the business loss.

Taking the business failure rate as the indicator, the deliberate attack method is adopted to attack each node one by one according to the descending order rank of the node importance of the node contraction algorithm, NTI and NCI[18]. Then the relationship between node failure and business loss is analyzed, and the result is shown in Figure 3.

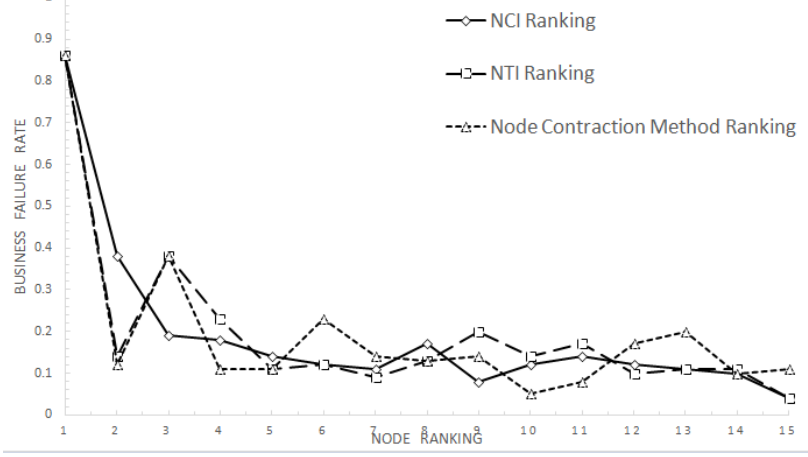

Figure. 3 Corresponding network business failure rates of three attacks

From the results of Figure 3, it is obvious that the polyline of business loss rate obtained by attacking each node according to the descending order ranking of NCI shows an overall decrease and the change range is not large. The business loss rate of network caused by the failure of node $v_{l}$ and node $v_{9}$ is 0.3 or more, while that of other nodes is less than 0.2 . The degree of the impact 
on the grid risk is relatively low, so the ranking of the node importance is relatively backward, which verifies the rationality of the NCI. The proposed NCI algorithm not only considers the business characteristics of the network load, but also well reflects the contribution of the node topology, so the NCI polyline in the figure is not strictly monotonic decreasing[19]. Taking nodes $v_{3}$ and $v_{7}$ as an example, the business failure rate caused by the failure of node $v_{7}$ is greater than that of node $v_{3}$. However, it can be seen from the topology diagram that the topological position of node $v_{3}$ is more important than that of node $v_{7}$. Combining the effects of the two nodes, the proposed NCI algorithm shows that node $v_{3}$ is more important than node $v_{7}$. The business failure rates of the other two attack modes in the figure are irregular. The main reason is that only the impact of the topology is considered, while the contribution of the network bearer business to the importance of the node is not considered. Therefore, analyzing the actual operation of the network has no practical significance.

\section{Conclusion}

In this paper, the change of the natural connectivity of the power communication network is calculated under the condition of node failure, and the Node Topology Importance (NTI) is defined; On the business level, the quantization method of the business importance of the power communication network is detailed firstly, and then the weighted adjacency matrix is obtained by integrating the type, importance and number of the business carried by the link. By combining the topology and the business characteristics, the eigenvalues of the adjacency matrix in the natural connectivity are replaced by the eigenvalues of the weighted adjacency matrix, and the Node Combining Importance (NCI) is defined. Based on the actual operation of the power communication network, and compared with other existing algorithms, the node importance can be calculated and the key nodes of the power communication network can be excavated by NCI more effectively and reasonably.

\section{Acknowledgments}

This work was financially supported by Project of State Grid Corporation of China in 2017; Research on Simulation Model and Simulation Method of Power Communication System (N0. XX71-17-006).

\section{References}

1. ANCILLOTTI E, BRUNO R, CONTI M. The role of communication systems in smart grids: Architectures, technical solutions and research challenges.J. Computer Communications, 36(1718):1665-1697, (2013).

2. Runze WU, Xiuyuan HU, Liangrui TANG. Node importance evaluation strategy based on triangle module applying to optical communication network for power grid.J. Journal of North China Electric Power University, 38(24): 28-32,(2011).

3. Qiongyao XIE, Changhong DENG, Hongsheng ZHAO, et al. Evaluation method for node importance of power grid based on the weighted network model.J. Automation of Electric Power Systems, 33(4): 21-24, (2009).

4. Ying ZENG, Ying WANG, Xijie DONG, et al. Node importance evaluation strategy on electric power communication backbone network.J. Journal of North China Electric Power University, 40(5): 65-69,(2013).

5. Runze WU, Baojian ZHANG, Liangrui TANG. A cascading failure based nodal importance evaluation method applied in dual network coupling model.J. Power System Technology, 39(4): 1053-1058,(2015).

6. Yuping RAO, Jingyu LIN, Deting HOU. Evaluation method for network invulnerability based on shortest route number.J. Journal of China Institute of Communications,30(4):113-117, (2009).

7. Jun WU, Suoyi TAN, YueJin TAN, et al. Analysis of invulnerability in complex networks based on natural connectivity.J. Complex Systems and Complexity Science,11(1):77-86,(2014).

8. Bing FAN, Liangrui TANG. Vulnerability analysis of power communication network.J. Proceeding of the CSEE, 34(7):1191-1197, (2014).

9. National Development and Reform Commission of the People's Republic of China. DL/T 5391-2007 Technical regulations of Power system communication design.S. Beijing: China Electric Power Press, (2007).

10. Dichen LIU, Xingpei JI, Bo WANG, et al. Topological vulnerability analysis and countermeasures of electrical communication network based on complex network theory.J. Power System Technology,39(12):3615-3621, (2015).

11. Yushen HOU, Xiuli WANG, Yue ZHANG, et al. Dimensional importance based Quasi-Monte Carlo method for power system reliability evaluation.J. Automation of Electric Power Systems,40(16):3137, (2016).

12. Yingjun XIE, Yuting WANG, Wei LI, et al. Indicator system for operation quality of electric power communication network.J. Electric Power, 50(10):22-27, (2017).

13. Li DI, Zheng ZHENG, Min XIA, et al. Node importance evaluation of electric power communication network based on fast density clustering.J. Power System Protection and Control, 44(13): 90-95, (2016).

14. Zihui GENG, Limin CUI, Qin SHU, et al. Crucial node decision algorithm based on TOPSIS algorithm in electric power communication network.J. Power System Protection and Control, 46(1): 78-86, (2018). 
15. JIANG K, DENG B. Risk evaluation method of electric power communication network.C.//Ninth International Conference on Natural Computation, IEEE, 1595-1599(2014).

16. Junchi JIA. Design of optical network capacity based on key link.J. Optical Communication Technology, 39(10):17-19(2015).

17. Yong CHEN, Aiqun HU, Xiao HU, et al. Evaluation method for node importance in communication networks.J. Journal of China Institute of Communications, 25(8):129134,(2005).

18. Yuejin TAN, Jun WU, Hongzhong DENG. Evaluation method for node importance based on node contraction in complex networks.J. Systems Engineering Theory\& Practice, 11(11):7983,(2006).

19. ESTRADA E, HATANO N, BENZI M. The physics of communicability in complex networks [J]. Phys Rep, 514(3):89-119,(2012). 